\title{
Riemann Surfaces, Clifford Algebras and Infinite Dimensional Groups
}

\author{
A. L. Carey ${ }^{1}$, M. G. Eastwood ${ }^{1}$ and K. C. Hannabuss ${ }^{2}$ \\ ${ }^{1}$ Department of Pure Mathematics, University of Adelaide, Adelaide, South Australia \\ ${ }^{2}$ Balliol College, Oxford OX1 3BJ England
}

\begin{abstract}
We introduce a class of Riemann surfaces which possess a fixed point free involution and line bundles over these surfaces with which we can associate an infinite dimensional Clifford algebra. Acting by automorphisms of this algebra is a "gauge" group of meromorphic functions on the Riemann surface. There is a natural Fock representation of the Clifford algebra and an associated projective representation of this group of meromorphic functions in close analogy with the construction of the basic representation of KacMoody algebras via a Fock representation of the Fermion algebra. In the genus one case we find a form of vertex operator construction which allows us to prove a version of the Boson-Fermion correspondence. These results are motivated by the analysis of soliton solutions of the Landau-Lifshitz equation and are rather distinct from recent developments in quantum field theory on Riemann surfaces.
\end{abstract}

\section{Introduction}

This note grew out of conversations between the first named author and John Palmer on applications of the results in [CP]. A cursory reading of that paper and the work of Date et al. [DJKM] reveals that the former should provide a rigorous framework for the latter. The main impediment to this program was to understand the intriguing phrase from [D]: "prepare fermions on an elliptic curve." In finding the recipe for this concoction we soon began to tread on ground already familiar from string theory, namely quantum field theory on Riemann surfaces. However it quickly became apparent that there were crucial differences.

Nevertheless in the early stages we benefitted greatly from lectures of D. Quillen and G. Segal given in Oxford in the spring of 1987 on string theory, Riemann surfaces and Bosonisation. While very similar ideas emerge here we find basic differences which can best be summarised by saying that we are dealing with the "real" case whereas the usual work on string theory deals with the complex case. As our account unwinds it will become clear that this involves more than just the usual distinction between Majorana and Dirac Fermions; nevertheless the same 
structures (infinite dimensional groups and vertex operators) arise and are crucial to our results.

This paper is not a definitive mathematical account of the work of Date et al. because we do not discuss in detail here the soliton solutions of the Landau-Lifshitz equation. In fact as our analysis developed it became clear that there were two distinct strands. The first was to understand the infinite dimensional groups (and representation theory) whose existence is suggested by [DJKM]. The second was to combine this with the ideas of Segal and Wilson [SW] to describe the solutions of the Landau-Lifshitz equation. Only the first is developed here as the details of the second are still to be fully understood. We hope to explore these in a later publication. Here we are concerned with the appropriate mathematical setting for the early part of [DJKM]. We present a general picture which encompasses a class of hyperelliptic curves ([DJKM] deal only with the genus one case) specialising to particular curves to connect up with $[\mathrm{CP}]$ and to elliptic curves to discuss vertex operators.

That this is a worthwhile project must stem in part from the fact that most mathematicians would find [DJKM] somewhat impenetrable owing to its daunting mixture of ingenius calculations and quantum field theory folklore. We have taken as our central theme the problem of constructing a representation of a central extension of a certain group of meromorphic functions on some Riemann surfaces which admit a fixed point free involution. These groups are the analogue for our context, of the loop groups which arise in string theory.

The discussion begins with the construction of line bundles over our Riemann surfaces $\mathscr{M}$. We consider a pair of generic points $z$ and $w$ and choose line bundles with divisor $g z-w$, where $g$ is the genus of $\mathscr{M}$. We show that the space of meromorphic sections of $L$ which are regular on a deleted neighbourhood of $\{z, w\}$ splits in a fashion which generalises the Hardy space splitting of functions on the circle. In Sect. 2 we introduce a non-degenerate symmetric bilinear form on this space of sections and construct the associated Clifford algebra. Associated with the splitting is a (Fock) representation of the Clifford algebra described in Sect. 3. Acting on the Clifford algebra as automorphisms is a group of meromorphic functions on $\mathscr{M}$. This group we show in Sect. 5 to be represented projectively on the representation space of the Clifford algebra. Section 4 is devoted to the case where $\mathscr{M}$ is hyperelliptic of a special form. In Sect. 6 we specialise to the genus 1 case the examples of Sect. 4 and introduce vertex operators and a weak form of the Boson-Fermion correspondence. This enables us to prove cyclicity of the projective representation of our group. Finally in Sect. 7 we indicate the anticipated connection between the representation theory of the groups studied here and the solutions of the Landau-Lifshitz equation. (This last section should be regarded as a report on work in progress and has been included as a result of a request by the referee.)

For the most part our notation on Riemann surfaces follows [G]:

$\vartheta(\zeta)$ : the divisor of the meromorphic function $\zeta$,

$\mathcal{O}(L)$ : the sheaf of germs of holomorphic cross sections of the line bundle $L$, $\Gamma(\mathscr{M}, \mathcal{O}(L))$ : holomorphic cross-sections of $L$, 
$H^{q}(\mathscr{M}, \mathcal{O}(L)): q^{\text {th }}$ cohomology group of $\mathscr{M}$ with coefficients in the sheaf $\mathcal{O}(L)$, $\gamma(L)$ : dimension of $\Gamma(\mathscr{M}, \mathcal{O}(L))$, $h^{1}(L)$ : dimension of $H^{1}(\mathscr{M}, \mathcal{O}(L))$, $c(L)$ : the Chern class of $L$, $\kappa:$ the canonical line bundle over the Riemann surface.

We assume the reader is familiar with the material in [G].

\section{Riemann Surfaces and Splittings}

Let $\mathscr{M}$ be a connected, compact Riemann surface of genus $g$. Choose two generic points on $\mathscr{M}$ say $z, w$. Let $L$ be a line bundle over $\mathscr{M}$ with divisor $m z+n w$ and $\mathscr{N}$ a neighbourhood of $\{z, w\}$ (strictly smaller than $\mathscr{M}$ ). A good example to keep in mind is the case where $\mathscr{N}$ is the disjoint union of two small discs centred on $z$ and $w$. (We actually specialise to this case in Sect. 4.) Now consider the MayerVietoris sequence for $\mathscr{M}=\mathscr{N} \cup(\mathscr{M}-\{z, w\})$ :

$$
\begin{aligned}
0 & \rightarrow \Gamma(\mathscr{M}, \mathcal{O}(L)) \rightarrow \Gamma(\mathscr{M}-\{z, w\}, \mathcal{O}(L)) \oplus \Gamma(\mathcal{N}, \mathcal{O}(L)) \\
& \rightarrow \Gamma(\mathscr{N}-\{z, w\}, \mathcal{O}(L)) \rightarrow H^{1}(\mathscr{M}, \mathcal{O}(L)) \rightarrow 0
\end{aligned}
$$

(the last arrow being a consequence of the fact that $\mathscr{N}$ and $\mathscr{M}-\{z, w\}$ are Stein). This defines a splitting

$$
\Gamma(\mathcal{N}-\{z, w\}, \mathcal{O}(L)) \cong \Gamma(\mathscr{M}-\{z, w\}, \mathcal{O}(L)) \oplus \Gamma(\mathcal{N}, \mathcal{O}(L)) / \Gamma(\mathscr{M}, \mathcal{O}(L))
$$

if and only if $H^{1}(\mathscr{M}, \mathcal{O}(L))=0$. This splitting will be useful to us provided $\Gamma(\mathscr{M}, \mathcal{O}(L))=0$ as well. Using Riemann-Roch:

$$
\begin{aligned}
\gamma(L) & =\gamma\left(\kappa L^{-1}\right)+c(L)+1-g \\
& =\gamma\left(\kappa L^{-1}\right)+m+n+1-g
\end{aligned}
$$

and Serre duality: $h^{1}(L)=\gamma\left(\kappa L^{-1}\right)$, so

$$
\gamma(L)-h^{1}(L)=c(L)+1-g=m+n+1-g .
$$

Thus to achieve our aim we need the conditions:

$$
\text { (i) } m+n+1-g=0 \text { and (ii) } \gamma(L)=0 \text {. }
$$

The first is satisfied if $m=g$ and $n=-1$ which means that $L$ comes equipped with a canonical section $\sigma$ with divisor $g z-w$. Then if $\gamma(L) \neq 0$ choose any non-zero holomorphic section $s$ of $L$ whence $s / \sigma$ is a meromorphic function with a zero at $w$ and a possible pole of order at most $g$ at $z$. For generic $z$ this cannot happen (i.e. for $z$ not a Weierstrass point) and so henceforth we assume $z$ and $w$ are generic. In particular, for the genus one case we can take for $z$ and $w$ any two distinct points because there are no meromorphic functions with just one pole.

We conclude that, by appropriate choice of $z, w$, the line bundle $L$ has divisor $g z-w$ and satisfies condition (ii) above so that we have a splitting:

$$
\Gamma(\mathcal{N}-\{z, w\}, \mathcal{O}(L)) \cong \Gamma(\mathscr{M}-\{z, w\}, \mathcal{O}(L)) \oplus \Gamma(\mathcal{N}, \mathcal{O}(L)) .
$$

Remark. Note that the genus zero case is a degenerate example where this splitting 
is the familiar Hardy one dividing meromorphic functions into those analytic inside and outside the unit disc. In this case $z$ is omitted $(g=0), w$ is the point zero and $\mathcal{N}$ is the complement of the point at infinity on the Riemann sphere.

\section{Clifford Algebras and Surfaces with Involution}

Choose a path $\gamma$ consisting of two small circles around $z$ and $w$ respectively, both traversed clockwise and lying in $\mathscr{N}$. We would like to define a symmetric complex bilinear form on $\Gamma(\mathcal{N}-\{z, w\}, \mathcal{O}(L))$ and hence a Clifford algebra. In fact we will restrict our attention to elements of $\Gamma(\mathcal{N}-\{z, w\}, \mathcal{O}(L))$ which extend to global meromorphic sections of $L$. We denote this space by $W$ and the corresponding subspaces of $\Gamma(\mathscr{M}-\{z, w\}, \mathcal{O}(L))$ and $\Gamma(\mathcal{N}, \mathcal{O}(L))$ by $W_{-}$and $W_{+}$respectively. Thus

$$
W=W_{-} \oplus W_{+} .
$$

Now Quillen and Segal have considered the case where $L$ is a spin structure (i.e. $\left.L^{2}=\kappa\right)$. We could try something similar as $c\left(L^{2}\right)=2(g-1)=c(\kappa)$, and hence one could define a complex bilinear form by taking two sections of $L$, tensoring them, and integrating around $\gamma$. This integration makes sense provided the divisor of the product is canonical, i.e. the tensor product is an Abelian differential. However this does not appear to lead naturally to automorphisms of the Clifford algebra defined by the complex orthogonal group. By constrast the latter group is forced on us if we suppose that $\mathscr{M}$ admits a fixed point free automorphism \# (as in [DJKM] for the genus one case) and use it in the construction of the Clifford algebra. This then leads us away from the string theory analogies and links up with the discussion in [DJKM].

Examples of surfaces with such involutions are the hyperelliptic curves associated with the algebraic equations

$$
\omega^{2}=\left(k^{2}-a_{1}^{2}\right)\left(k^{2}-a_{2}^{2}\right) \cdots\left(k^{2}-a_{g+1}^{2}\right),
$$

where $g$ is odd and $a_{1}, a_{2}, \ldots, a_{g}$ are distinct non-zero complex numbers. Here the involution is given by $(\omega, k)^{\#}=(-\omega,-k)$. (In the even case this involution has fixed points.) Assume for the moment $\mathscr{M}$ is the surface associated with this equation. Given a line bundle $L$ over $\mathscr{M}$ satisfying conditions (i) and (ii) of the previous section we choose $z$ and $w$ to be the two points at $\infty$ in the coordinates $k, \omega$ or in other words the two points lying over the point at $\infty$ on the Riemann sphere under the covering map $(\omega, k) \rightarrow k$. These points are generic (i.e. not Weierstrass, the Weierstrass points being the $\pm a_{i}$ 's). We denote these points by $\infty_{-}(=z)$ and $\infty_{+}\left(=z^{\#}\right)$ (this notation accords with the fact that $\lim \omega / k^{g+1}$ is \pm 1 at $\infty_{ \pm}$). Notice that $\infty_{-}^{\#}=\infty_{+}$. These surfaces will form our main examples in what follows.

Some features of this example are generic. To see this we note the following fact (see also [C] for a more general argument).

Proposition 2.1. Suppose $\mathscr{M}$ (a Riemann surface of genus $g$ ) is a double covering of $\mathscr{M}_{1}$ (with genus $g_{1}$ ) ramified at $r$ points. Then $2 g=4 g_{1}+r-2$. 
This is proved by triangulating $\mathscr{M}_{1}$ so that the $r$ points of the ramification are amongst the vertices. Suppose this triangulation has $v$ vertices, $e$ edges and $f$ faces. Pulling back to $\mathscr{M}$ gives a triangulation with $v^{\prime}$ vertices, $e^{\prime}$ edges and $f^{\prime}$ faces, where $f^{\prime}=2 f, e^{\prime}=2 e$ and $v^{\prime}=2 v-r$. Thus comparing Euler characteristics

$$
f^{\prime}-e^{\prime}+v^{\prime}=2(f-e+v)-r .
$$

But the Euler characteristic of $\mathscr{M}_{1}$ is $2-2 g_{1}$ and that of $\mathscr{M}$ is $2-2 g$, hence the result.

Corollary 2.2. Suppose $\mathscr{M}$ is a Riemann surface of genus $g$. If $\mathscr{M}$ has a fixed point free involution \# then $g$ is odd and then $\mathscr{M} / \#$ has genus $(g+1) / 2$.

Returning to our main theme, recall that we would like to construct a symmetric bilinear form on the space $W$. To this end suppose that $L$ is any line bundle over $\mathscr{M}$ satisfying the conditions

$$
\text { (i) } c(L)=g-1 \text { and (ii) } \gamma(L)=0
$$

as above. Suppose also that $L \otimes L^{\#}=\kappa$. Topologically this is always the case as both sides have Chern class $2 g-2$ but analytically this is a very strong condition. In any case assume this holds, choose $d P$ to be a holomorphic section of $L^{*} \otimes L^{\# *} \otimes \kappa$ and take $z=w^{\#}$. Then define a complex bilinear form on $W$ $(=\Gamma(\mathcal{N}-\{z, w\}, \mathcal{O}(L)))$ by

$$
(\phi, \psi)=\int_{\gamma} \phi \psi^{\#} d P
$$

for $\phi$ and $\psi$ in $W$, where $\gamma$ is a closed path in $\mathscr{N}-\{z, w\}$ encircling both $z$ and $w$ once. We would like this form to be symmetric and it is easy to see that it is either symmetric or skew as follows.

Our assumption that $L^{*} \otimes L^{\# *} \otimes \kappa$ is trivial means that the space of holomorphic sections of $L^{*} \otimes L^{\# *} \otimes \kappa$ is one dimensional. Moreover \# acts on this vector space with $\#^{2}=1$ whence $d P$ must satisfy one of

(a) $\# d P=d P$ in which case (,) is symmetric,

(b) $\# d P=-d P$ in which case (,) is skew.

If \# has fixed points then (b) is forced. To see this choose a local coordinate $t$ centred on the fixed point so that $\# t=-t$. Now choose any meromorphic section $\varphi$ of $L$ with no zero or pole at the fixed point. In the local co-ordinate $t$

$$
\varphi \varphi^{\#} d P=f(t) d t,
$$

where $f(0) \neq 0$ (note: $d P$ has no zeros). Applying \# to both sides of this equation gives

$$
\varphi \varphi^{\#} d P^{\#}=(f(t) d t)^{\#}=-f(-t) d t
$$

and setting $t=0$ implies that $d P(0)=-d P^{\#}(0)$ so case (b) follows.

Notice that this argument is carried out under the assumptions: (a) $z^{\#}=w$, (b) $L \otimes L^{\#} \cong \kappa$. If (a) is not satisfied (as might be the case for even $g$ in the hyperelliptic curve example) then our discussion needs further elaboration which 
we do not give here (we are mainly interested in the generic case where special choices of divisor are not made). In the case of assumption (b) it is far from clear that such an $L$ can be found. All we have shown is that if we want a symmetric bilinear form then the involution \# must be fixed point free. However if the latter holds then it is easy to find such a bundle $L$ since we can take any square root of the canonical bundle on $\mathscr{M} / \#$ and pull it back to $\mathscr{M}$. In our explicit example (2.1) we can take $L$ to be the bundle arising from the divisor $g z-w$ as then $L \otimes L^{\#}$ arises from the divisor $(g-1) z+(g-1) w$ and we may take $d P$ to correspond to $d k / \omega$ (an Abelian differential with a zero of order $g-1$ at $\left.\infty_{ \pm}\right)$. It is evident that $\# d P=d P$ in this case. More generally a suitable $d P$ may be manufactured from any Abelian differential on $\mathscr{M} / \#$. For simplicity we will restrict attention, for the rest of this article, to the case of hyperelliptic curves of the form (2.1). This is no restriction in the genus one case and while there may be other examples there seems no point in pursuing such generality here. Finally we choose the orientation of $\gamma$ to be clockwise about $\infty_{ \pm}$.

It is useful at this point to make an identification of our space of sections, $W$, with a function space. Firstly, $L$ comes equipped with a canonical section which is holomorphic on $\mathscr{M}-\{z, w\}$ and which has divisor $g z-w$ on $\mathscr{N}$. Then elements of $W_{-}$may be regarded as functions analytic on $\mathscr{M}-\{z, w\}$ with (possibly) poles at the deleted points, while sections in $W_{+}$can be identified with functions which are meromorphic on $\mathscr{M}$, analytic in $\mathscr{N}$ except possibly at $z$ where they may have a pole of order no greater than $g$ and which must have a zero at $w$. The elements of $W$ are thus identified with meromorphic functions $\mathscr{M}$ which are analytic in $\mathscr{N}-\{z, w\}$.

Lemma 2.3. The bilinear form on $W$ is nondegenerate and symmetric.

The proof of non-degeneracy in Lemma 2.3 and indeed much of our subsequent analysis depends on the existence of meromorphic functions with prescribed divisors. To prove that these functions exist is our next task.

Proposition 2.4. Given two distinct points $z_{0}, w_{0}$ in $\mathscr{M}$ and a neighbourhood $\mathscr{U}$ of $z_{0}$ there is a meromorphic function on $\mathscr{M}$ with all its poles at $z_{0}$ and one zero at $w_{0}$ and all other zeros in $\mathscr{U}$.

Proof. Let $J(\mathscr{M})$ be the Jacobi variety of $\mathscr{M}$ and define $j: \mathscr{M} \rightarrow J(\mathscr{M})$ in the usual way by choosing a basis of the space of sections of the canonical bundle, say $\omega_{1}, \ldots, \omega_{g}$ and defining $j(x)$ to be the point in $J(\mathscr{M})$ determined by the $g$ complex numbers

$$
\int_{z_{0}}^{x} \omega_{i}
$$

Jacobi inversion tells us that the symmetrised product $\mathscr{S}_{g} \mathscr{M}$ (i.e. $\mathscr{M} x \mathscr{M} x \cdots x \mathscr{M}$ ( $g$ times) modulo the action of the symmetric group) maps onto $J(\mathscr{M})$ under the map which sends $\left(p_{1}, p_{2}, \ldots, p_{g}\right)$ to $j\left(p_{1}\right)+j\left(p_{2}\right)+\cdots+j\left(p_{g}\right)$. Now $\mathscr{S}_{g} \mathscr{U}$ has a neighbourhood of zero in $J(\mathscr{M})$ as its image under this map. As there is a positive integer $m$ such that $-j\left(w_{0}\right) / m$ lies in this neighbourhood we can assert the existence of points $p_{1}, p_{2}, \ldots, p_{g}$ with $j\left(p_{1}\right)+j\left(p_{2}\right)+\cdots+j\left(p_{g}\right)=-j\left(w_{0}\right) / m$. It follows from 
this and the fact that $j\left(z_{0}\right)=0$ that there is a meromorphic function on $\mathscr{M}$ with divisor

$$
w_{0}+m p_{1}+m p_{2}+\cdots+m p_{g}-(g m+1) z_{0} .
$$

Corollary 2.5. There is a meromorphic function on $\mathscr{M}$ with divisor

$$
\begin{aligned}
& w_{0}+m p_{1}+m p_{2}+\cdots+m p_{g}-\left(w_{0}^{\#}+m p_{1}^{\#}+m p_{2}^{\#}+\cdots+m p_{g}^{\#}\right) \\
& -(g m+1) z_{0}+(g m+1) z_{0}^{\#} .
\end{aligned}
$$

The function required here is obtained by taking the function whose existence is proved in the proposition say $\phi_{0}$ and taking the ratio $\phi_{0} / \phi_{0}^{\#}$, where $\phi_{0}^{\#}$ is defined by $\phi_{0}^{\#}(x)=\phi_{0}\left(x^{\#}\right)$.

Now we return to the proof of Lemma 2.3. Suppose $(\phi, \psi)=0$ for all $\psi$ in $W$. If $q_{1}, q_{2}, \ldots, q_{m}$ are the poles of $\phi$ then we can find for each $i=1, \ldots, m$, by Proposition 2.4, a meromorphic function $\phi_{i}$ with a simple zero at $q_{i}$, all its poles at $z$, and all other zeros in $\mathscr{N}$. Then $\phi_{1} \phi_{2} \cdots \phi_{m} \phi$ is regular outside $\mathscr{N}$ and $\left(\phi_{1} \phi_{2} \cdots \phi_{m} \phi, \psi\right)=0$ for all $\psi$ in $W$. Another application of Proposition 2.4 gives us a function $\phi_{0}$ whose divisor on $\mathcal{N}$ is $z$. Then multiplying $\phi_{1} \phi_{2} \cdots \phi_{m} \phi$ by suitable powers of $\phi_{0}$ and of $\phi_{0}^{\#}$ we can arrange that the product $\phi_{0}^{r}\left(\phi_{0}^{\#}\right)^{s} \phi_{1} \phi_{2} \cdots \phi_{m} \phi \psi^{\#} d P$ has a simple pole at $z$ and is regular elsewhere in $\mathcal{A}$. Integrating this around $\gamma$ cannot give zero which is a contradiction. So our bilinear form is non-degenerate proving the lemma.

Remark 2.6. The surfaces with fixed point free involutions are certainly more ubiquitous than the hyperelliptic curve examples (2.1). (We thank Roger Richardson for pointing out the following argument.) We saw above that $\mathscr{M} / \#$ has genus $(g+1) / 2$ and the moduli space of all Riemann surfaces with this genus has dimension $(3 g-3) / 2$. Now the covering $\mathscr{M} \rightarrow \mathscr{M} / \#$ is determined by an index two subgroup of $\pi_{1}(\mathscr{M} / \#)$ so it follows that the moduli space of pairs $(\mathscr{M}$,\#) has dimension $\geqq(3 g-3) / 2$ and for sufficiently large $g$ the latter is clearly greater than the number of possibilities given by the hyperelliptic curves (2.1). Actually stronger information on this situation may be found in [C].

We now introduce the two objects of greatest interest to us. The first is the group $\mathscr{G}$ which consists of functions $\phi$ meromorphic on $\mathscr{M}$ and analytic and non-vanishing in $\mathscr{N}-\{z, w\}$ and which satisfy $\phi^{\#}=\phi^{-1}$. The second is the algebraic Clifford algebra $\mathscr{C}(W)$ over $W$ defined by the symmetric bilinear form given above. The latter is the associative algebra with identity generated by the elements $c(w)$ where $w$ lies in $W$, subject to the usual Clifford algebra relations

$$
c\left(w_{1}\right) c\left(w_{2}\right)+c\left(w_{2}\right) c\left(w_{1}\right)=\left(w_{1}, w_{2}\right) I,
$$

where $I$ denotes the identity. Notice that $\mathscr{G}$ acts by automorphisms of $\mathscr{C}(W)$ via its action by multiplication operators on $W$, i.e. $w \rightarrow \phi w$.

Note that we could equally consider an $n$ component version of this construction in which $\mathscr{G}$ is replaced by matrix valued meromprophic functions on $\mathscr{M}$ which are analytic in $\mathscr{N}-\{z, w\}$, and on restriction to the latter take their values in $G L(n)$ and are subject to the constraint $\phi^{\#}=\left(\phi^{-1}\right)^{\tau}$, where $\tau$ denotes the matrix transpose. 
To see that $\mathscr{G}$ is non-empty, let $K$ be a meromorphic function whose divisor is given by Corollary 2.5 with $w_{0}=z^{\#}$ and with $p_{1}, p_{2}, \ldots, p_{g}, z_{0}$ and their images under \# all outside $\mathscr{N}$. In fact by construction $K^{\#} K=1$, and hence $K$ is in $\mathscr{G}$.

\section{The Fock Representation of $\mathscr{C}(W)$}

Associated with any splitting of $W$ into subspaces isotropic with respect to the bilinear form is a representation. Since $W=W_{+} \oplus W_{-}$is such a splitting we will now construct this representation referring to it in the future as the Fock representation. The space on which this representation acts is the antisymmetric tensor algebra over $W_{+}$written $\wedge W_{+}$.

Now we define the action of $\mathscr{C}(W)$ on $\wedge W_{+}$. For $w_{+}$in $W_{+}$, let $F\left(w_{+}\right)$be the operator:

$$
F\left(w_{+}\right) w_{1} \wedge w_{2} \wedge w_{3} \wedge \cdots \wedge w_{n}=w_{+} \wedge w_{1} \wedge w_{2} \wedge \cdots \wedge w_{n} .
$$

If $w_{-}$is in $W_{-}$then we define $F\left(w_{-}\right)$to act by

$$
F\left(w_{-}\right) w_{1} \wedge w_{2} \wedge w_{3} \wedge \cdots \wedge w_{n}=\Sigma_{j}(-1)^{j-1}\left(w_{-}, w_{j}\right) w_{1} \wedge w_{2} \wedge \cdots \hat{w}_{j} \wedge \cdots \wedge w_{n} .
$$

Then it is straightforward to check that $F$, defined by

$$
F(w)=F\left(w_{-}\right)+F\left(w_{+}\right),
$$

where $w$ is in $W$ and $w_{ \pm}$is in $W_{ \pm}$is a representation of $\mathscr{C}(W)$. We use the notation $\Omega$ for the vector $1 \oplus 0 \oplus 0 \oplus 0 \oplus \cdots$.

Note that the usual convention in physics is to introduce the operator $N$ on $\wedge W_{+}$defined by

$$
N w_{1} \wedge w_{2} \wedge w_{3} \wedge \cdots \wedge w_{n}=n w_{1} \wedge w_{2} \wedge w_{3} \wedge \cdots \wedge w_{n},
$$

and hence the operators

$$
c\left(w_{-}\right)=N^{-1 / 2} F\left(w_{-}\right) \text {and } c\left(w_{+}\right)=(N+1)^{1 / 2} F\left(w_{+}\right) .
$$

With this convention one also obtains a representation of $\mathscr{C}(W)$ which is compatible with the usual Hilbert space structure imposed on $\wedge W_{+}$. We shall have more to say about this in Sect. 4. For our purposes it is not important which way the representation of $\mathscr{C}(W)$ is defined.

Our eventual aim is to construct a projective representation of $\mathscr{G}$ on $\wedge W_{+}$. To motivate our approach to this problem it is useful to link up with the literature on representations of other infinite dimensional groups. Hence we suppose for the moment that $W$ is a Hilbert space with a distinguished conjugation $\Gamma$ such that the inner product on $W$, written $<,>$ and the complex bilinear form (,), are related by $\langle.,\rangle=.(\Gamma$.,.). Then the complex orthogonal group consists of bounded operators on $W$ which satisfy $\Gamma G^{*} \Gamma=G^{-1}$. Given a splitting of $W$ into a direct sum of Hilbert subspaces $W_{+}$and $W_{-}$isotropic with respect to (,) we introduce the projections $Q_{ \pm}$onto these subspaces. We shall suppose that $\Gamma W_{+}=W_{-}$and define the operator $Q=Q_{+}-Q_{-}$. Then the restricted complex orthogonal group consists of complex orthogonals $G$ such that $Q G-G Q$ is Hilbert-Schmidt. This is a topological group where we impose the norm topology on the operators 
$Q G+G Q$ and the Hilbert-Schmidt topology on the operators $Q G-G Q$. The dense subgroup consisting of $G$ such that $Q G-G Q$ is finite rank we write $\mathcal{O}_{f}$. This group as well as the restricted complex orthogonal group has two connected components (see $\left[\mathrm{CO}^{\prime} \mathrm{B}\right]$ and $[\mathrm{CP}]$ ) labelled by the index map

$$
\iota: G \rightarrow \operatorname{dim} \operatorname{ker} Q_{-} G Q_{-} \quad(\bmod 2),
$$

where by kernel we mean the kernel in $W_{-}$.

The Hilbert-Schmidt restriction is exactly what is needed to enable the construction of a projective representation of the restricted orthogonal group on a dense invariant subspace of $\wedge W_{+}$by unbounded (invertible) operators. For the details of its construction we refer the reader to $[\mathrm{CP}]$. We shall not use this argument to construct our representation of $\mathscr{G}$ preferring instead a direct method. However, we shall indicate below how this Hilbert space approach works.

On restriction to the subgroup of the restricted orthogonal group consisting of unitary operators on $W$ (i.e. those commuting with $\Gamma$ ) one obtains a projective representation by unitary operators on $\wedge W_{+}$. As a representation of the connected component of the identity of this subgroup, $\wedge W_{+}$decomposes into two irreducible invariant subspaces spanned by the odd and even tensor powers of $W_{+}$. Elements $G$ of the restricted orthogonal group with $l(G)=1$ map between these subspaces. Hence if one is aiming to produce a cyclic projective representation of $\mathscr{G}$ it is necessary to exhibit elements of the group which satisfy $l(G)=1$.

Now return to our situation in which $W$ is not a Hilbert space. It still makes sense to introduce the group $\mathcal{O}_{f}$ consisting of complex orthogonals $G$ for which $Q G-G Q$ is finite rank. We shall now show that $K$ is an element of $\mathcal{O}_{f}$. (We will not discuss the conjugation $\Gamma$ in this section.) More precisely we shall prove

Proposition 3.1. The group $\mathscr{G}$ is a subgroup of $\mathcal{O}_{f}$.

This result is a consequence of the more general following fact.

Proposition 3.2. Let $\mathscr{M}$ be a compact Riemann surface of genus $g$ and let $L$ be a line bundle over $\mathscr{M}$ satisfying conditions (i) and (ii) of Sect. 2. Let $\varphi$ be an arbitrary meromorphic function on $\mathscr{M}$ then $Q_{+} \varphi Q_{-}$and $Q_{-} \varphi Q_{+}$are finite rank.

Proof. Choose any global meromorphic section $\sigma$ of $L$ and write its divisor as

$$
\vartheta(\sigma)=\lambda_{1}+\lambda_{2}+\lambda_{3},
$$

where $\lambda_{1}$ is the divisor on $\{z, w\}, \lambda_{2}$ is the divisor on $\mathscr{N}-\{z, w\}$ and $\lambda_{3}$ is the divisor on $\mathscr{M}-\mathscr{N}$. A local section $s$ of $L$ gives rise to a local meromorphic function $s / \sigma$ and so the splitting of $W$ into $W_{-}$and $W_{+}$may be regarded as imposing the following constraints on a meromorphic function $f$ on $\mathscr{M}$ :

$$
\begin{aligned}
W & =\left\{f \mid \vartheta(f) \geqq-\lambda_{2} \text { on the subset } \mathscr{N}-\{z, w\}\right\}, \\
W_{+} & =\left\{f \mid \vartheta(f) \geqq-\lambda_{1}-\lambda_{2} \text { on the subset } \mathscr{N}\right\}, \\
W_{-} & =\left\{f \mid \vartheta(f) \geqq-\lambda_{2}-\lambda_{3} \text { on the subset } \mathscr{M}-\{z, w\}\right\} .
\end{aligned}
$$

Given a general $f$ in $W$ we write its divisor as

$$
\vartheta(f)=\tau_{1}+\tau_{2}+\tau_{3}
$$


where, again, this decomposition corresponds to the decomposition of $\mathscr{M}$ into the disjoint sets $\{z, w\}, \mathscr{N}-\{z, w\}$ and $\mathscr{M}-\mathscr{N}$ respectively. The only restriction is $\tau_{2} \geqq-\lambda_{2}$. If we now split $f$ as a sum $f_{+}+f_{-}$of its components in $W_{+}$and $W_{-}$ respectively then a little thought shows we must have

$$
\begin{aligned}
& \vartheta\left(f_{+}\right) \geqq-\lambda_{1}-\lambda_{2}+\min \left\{-\lambda_{3}, \tau_{3}\right\}, \\
& \vartheta\left(f_{-}\right)=-\lambda_{2}-\lambda_{3}+\min \left\{-\lambda_{1}, \tau_{1}\right\} .
\end{aligned}
$$

There is a similar decomposition of the divisor of $\varphi$ :

$$
\vartheta(\varphi)=\mu_{1}+\mu_{2}+\mu_{3} .
$$

Now we consider the image of an $f$ in $W_{-}$under $Q_{+} \varphi Q_{-}$. With the notation of (3.1) we have

and

$$
\tau_{2}+\tau_{3} \geqq-\lambda_{2}-\lambda_{3}
$$

$$
\vartheta(\varphi f)=\left(\mu_{1}+\tau_{1}\right)+\left(\mu_{2}+\tau_{2}\right)+\left(\mu_{3}+\tau_{3}\right)
$$

and hence, by (3.2)

$$
\vartheta\left((\varphi f)_{+}\right) \geqq-\lambda_{1}-\lambda_{2}+\min \left\{-\lambda_{3}, \mu_{3}+\tau_{3}\right\} .
$$

But as $f$ is in $W_{-}$the constraint, given above, on its divisor implies $\tau_{3} \geqq-\lambda_{3}$ and so

$$
\vartheta\left((\varphi f)_{+}\right) \geqq-\lambda_{1}-\lambda_{2}+\min \left\{-\lambda_{3}, \mu_{3}-\lambda_{3}\right\} .
$$

Hence $(\varphi f)_{+}$may be regarded as a holomorphic section of the line bundle corresponding to the divisor

$$
\lambda_{1}+\lambda_{2}+\max \left\{\lambda_{3},-\mu_{3}+\lambda_{3}\right\}
$$

whence the dimension of the space of such sections provides a bound for the rank of $Q_{+} \varphi Q_{-}$. Similarly, the rank of $Q_{-} \varphi Q_{+}$is bounded by the dimension of the space of holomorphic sections of the line bundle corresponding to the divisor

$$
\lambda_{2}+\lambda_{3}+\max \left\{\lambda_{1},-\mu_{1}+\lambda_{1}\right\} \text {. }
$$

Remark 3.3. The above argument is more general than it may appear. In fact it works when $L$ is a line bundle with divisor $z_{1}+z_{2}+\cdots+z_{g}-w$ (for a generic choice of these points with $\mathscr{N}$ an open set containing them).

Remark 3.4. One may also use this argument to establish the matrix analogue of Proposition 3.1. Here one tensors an $n$-dimensional complex vector space onto $L$, appropriately generalises the bilinear form and considers the group of $n \times n$ matrix valued meromorphic functions on $\mathscr{M}$ which are holomorphic on $\mathscr{N}-\{z, w\}$ and satisfy $\varphi^{\#}=\left(\varphi^{-1}\right)^{\tau}$, where $\tau$ denotes the matrix transpose. Then this group also has the property that it is a subgroup of $\mathcal{O}_{f}$.

\section{A Special Case}

In this section we assume $\mathscr{M}$ is the hyperelliptic curve determined by the equation:

$$
\omega^{2}=\left(k^{g+1}-a^{g+1}\right)\left(k^{g+1}-b^{g+1}\right)
$$


where $a$ and $b$ are fixed distinct non-zero complex numbers. Then as before we choose $z=\infty_{\ldots}$. In this case one may write down explicit elements of $\mathscr{G}$ such as the function $h$ defined by:

$$
h(\omega, k)=\left(\omega+k^{g+1}-a^{g+1}\right) /\left(\omega-k^{g+1}+a^{g+1}\right) .
$$

Then it is immediate that $h h^{\#}=1$ and so $h$ is in $\mathscr{G}$. One easily checks that $h$ has divisor $(g+1) \infty_{-}-(g+1) \infty_{+}$. To construct more interesting elements of $\mathscr{G}$ recall Riemann-Roch:

$$
\gamma\left(L_{1}\right)=\gamma\left(\kappa L_{1}^{-1}\right)+c\left(L_{1}\right)+1-g .
$$

To guarantee the existence of a holomorphic section of $L_{1}$ we need $c\left(L_{1}\right) \geqq g+1$. This implies one may specify generically $g+1$ poles for any meromorphic function. By adding a constant one may also specify one of its $g+1$ zeros. Choose $q$ generically in $\mathscr{M}$ and let $\zeta$ be any meromorphic function with divisor

$$
\vartheta(\zeta)=p_{1}+p_{2}+\cdots+p_{g}+\infty_{-}-(g-1) \infty_{+}-q-q^{\#} .
$$

Then

$$
\vartheta\left(\zeta^{\#}\right)=p_{1}^{\#}+p_{2}^{\#}+\cdots+p_{g}^{\#}+\infty_{+}-(g-1) \infty_{-}-q-q^{\#} .
$$

Now define $K_{0}=\zeta / \zeta^{\#}$ so that trivially $K_{0} K_{0}^{\#}=1$ and

$$
\vartheta\left(K_{0}\right)=p_{1}+p_{2}+\cdots+p_{g}-p_{1}^{\#}-p_{2}^{\#}-\cdots-p_{g}^{\#}+g \infty_{-}-g \infty_{+} .
$$

By choosing $\mathscr{N}$ so that $p_{1}, p_{2}, \ldots, p_{g}$ lie outside it we find that $K_{0}$ is in $\mathscr{G}$. Now the function $K_{0} h^{\#}$ has divisor

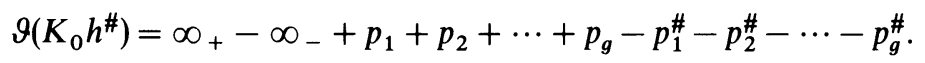

An argument similar to that in the proof of Proposition 3.2 shows that $K_{0} h^{\#}$ has the property that the kernel of $Q_{-} K_{0} h^{\#} Q_{-}$as an operator on $W_{-}$and of $Q_{+} K_{0} h^{\#} Q_{+}$as an operator on $W_{+}$are both one dimensional. To see this consider first the case where $f$ is in $W_{-}$and in the kernel of $Q_{-} K_{0} h^{\#} Q_{-}$. Then $K_{0} h^{\#} f$ has to lie in $W_{+}$but this is impossible unless $\vartheta(f) \geqq-(g-1) \infty_{-}$, which means $f$ is constant. On the other hand if $f$ is in the kernel of $Q_{+} K_{0} h^{\#} Q_{+}$then $K_{0} h^{\#} f$ lies in $W_{-}$and so

$$
\vartheta\left(K_{0} h^{\#} f\right) \geqq 2 \infty_{+}-(g+1) \infty_{-} .
$$

But then $\vartheta\left(K_{0} f\right) \geqq-(g-1) \infty_{+}$which forces $K_{0} f$ to be constant. So the kernel is spanned by $K_{0}^{\# \text {. }}$

For this example we can make contact with the Hilbert space formalism of $[\mathrm{CP}]$. This is done by introducing the functions $\alpha_{r}$ and $\beta_{r}$ defined for integral $r$ by

$$
\alpha_{r}(k, \omega)=k^{-r-1}, \quad \beta_{r}(k, \omega)=h^{\#}(k, \omega)(-k)^{r} .
$$

Then one may check that the following relations hold:

$$
\left(\alpha_{r}, \alpha_{s}\right)=0, \quad\left(\alpha_{r}, \beta_{s}\right)=c_{r} \delta_{r s}, \quad\left(\beta_{r}, \beta_{s}\right)=0,
$$

where $c_{r}$ is some non-zero constant. To see this note that in all cases except where we pair $\alpha$ 's and $\beta$ 's with $r=s$ either the integrand is regular in the neighbourhood 
$\mathscr{N}$ or the integrand is regular outside the contour. Only in the case where we pair $\alpha$ 's and $\beta$ 's and $r=s$ does the integrand have a simple pole at $\infty_{+}$.

Now assume we have renormalised $\beta_{s}$ so that $\left(\alpha_{r}, \beta_{s}\right)=\delta_{r s}$. Then we can inject $W$ into $L^{2}\left(S^{1}, \mathbb{C}^{2}\right)$ via the map which sends the pair $\alpha_{r}, \beta_{s}$ to the $\mathbb{C}^{2}$ valued function $\theta \rightarrow\left(e^{i r \theta}, e^{i s \theta}\right) /(2 \pi)^{1 / 2}$. It is not immediately clear that every function in $W$ can be written in the appropriate form for this map to be everywhere defined (i.e. as $a$, possibly infinite, linear combination of the $\alpha$ 's and $\beta$ 's). However, since

$$
h(\omega, k)=\left(2 \omega+2 k^{g+1}-a^{g+1}-b^{g+1}\right) /\left(a^{g+1}-b^{g+1}\right),
$$

it suffices to show that every function in $W$ may be written as $f_{1}(k)+\omega f_{2}(k)$, where $f_{1}$ and $f_{2}$ are the Laurent series of functions regular on the image of $\mathscr{N}$ in the Riemann sphere except possibly at $\infty$. Now since any meromorphic function on $\mathscr{M}$ is a rational function of $k$ and $\omega$ it suffices to establish the claim for functions of the form $(p(k)+\omega q(k))^{-1}$, where $p$ and $q$ are polynomials. By multiplying by a suitably large inverse power of $k$ we can assume that this function is regular in $\mathscr{N}$. We can assume without loss that $\mathscr{N}$ is a pair of discs which include the unit circle (in the $k$ variable) and is \# invariant, so the function $(p(k)-\omega q(k))^{-1}$ is also regular in $\mathscr{N}$. It fallows that the function $(p(k)-\omega q(k)) /\left(p(k)^{2}-\omega^{2} q(k)^{2}\right)$ shares this property and since the denominator is a polynomial it has a convergent Taylor expansion in powers of $k^{-1}$ in $\mathscr{N}$. This establishes the claim.

Note that the bilinear form also maps over to $L^{2}\left(S^{1}, \mathbb{C}^{2}\right)$ under this map. Using the Fourier transform we identify the latter space with pairs of sequences in which case the bilinear form goes over to the map which sends two pairs of sequences $\left(f_{r}\right),\left(g_{r}\right)$ and $\left(f_{r}^{\prime}\right),\left(g_{r}^{\prime}\right)$ to $\Sigma_{r}\left(f_{r} g_{r}^{\prime}+g_{r} f_{r}^{\prime}\right)$. The bilinear form and the inner product $<.,$.$\rangle are then related by \langle.,\rangle=.(\Gamma$.,.), where $\Gamma$ is the conjugation which sends the pair of sequences $\left(\left(f_{r}\right),\left(g_{r}\right)\right)$ to the pair $\left(\left(\bar{g}_{r}\right),\left(\bar{f}_{r}\right)\right)$ (here the bar denotes complex conjugation). Pulling this conjugation back to $W$ it has the form

$$
\Gamma\left(\Sigma_{r}\left(f_{r} \alpha_{r}+g_{r} \beta_{r}\right)\right)=\Sigma_{r}\left(\bar{g}_{r} \alpha_{r}+\bar{f}_{r} \beta_{r}\right) .
$$

Using this we define the obvious pre-Hilbert space structure on $W$. This has the great advantage that now we are in a position to import the Hilbert space theory of [CP]. It has the flaw that this complex conjugation appears to be very unnatural form the geometric viewpoint we have adopted to this point. We see no way to give an intrinsic geometric definition for the conjugation.

If one now completes $W$ to give a Hilbert space $H$ then $W_{ \pm}$close up to give subspaces with precise regularity properties. As the functions $\alpha_{r}$ for $r<0$ and $\beta_{r}$ for $r \geqq 0$ lie in $W_{-}$while $\alpha_{r}$ for $r \geqq 0$ and $\beta_{r}$ for $r<0$ lie in $W_{+}$, it follows from the embedding in $L^{2}\left(S^{1}, \mathbb{C}^{2}\right)$ that we may identify the closure of $W_{-}$with the direct sum of two copies of the Hardy space consisting of functions regular outside the unit circle and $L^{2}$ on the boundary. Then $W_{+}$is the direct sum of two copies of the orthogonal complement.

Now we have to check that our group $\mathscr{G}$ lies in $\mathcal{O}_{f}$ whether we define the latter with respect to operators on $W$ or on its closure. But, for example, for any $\varphi$ in $\mathscr{G}$ we have $Q_{+} \varphi W_{-}$a finite dimensional subspace and since such spaces are closed in $H$ it follows that $Q_{+} \varphi Q_{-} H$ is also finite dimensional. Notice that because of the regularity properties of the completions of $W_{ \pm}$which we found in the preceding 
paragraph the kernel dimensions of $Q_{-} K_{0} h^{\#} Q_{-}$and $Q_{+} K_{0} h^{\#} Q_{+}$do not change on passing from $W$ to $H$. Thus we need not worry about the fact that we have completed $W$. Now there is a topology on $\mathscr{G}$ with respect to which we may discuss the continuity properties of the index map. Note that this begs the question of the topological structure of $\mathscr{G}$ in some natural function space topology. We have not investigated the latter.

Our first side effect of these constructions is an index formula. Suppose $\phi$ is any element of $\mathscr{G}$. Then $\phi$ has a pole and a zero of the same order at the two points at infinity. By multiplying by a suitable power of $K_{0} h^{\#}$ one then obtains a function $\phi^{\prime}$ in $\mathscr{G}$ which is regular in $\mathscr{N}$ and hence has no zeros or poles in that neighbourhood. For any function $f$ in $W_{-}, \phi^{\prime} f$ cannot be in $W_{+}$because $\phi^{\prime}$ cannot change the divisor at the points at infinity. Similarly if $f$ is in $W_{+}$then $\phi^{\prime} f$ remains in $W_{+}$. Hence the kernel of $Q_{-} \phi^{\prime} Q_{-}$as an operator on $W_{-}$must be zero and similarly with the kernel of $Q_{+} \phi^{\prime} Q_{+}$. Notice that in completing $W$ to $H$ this argument still works because of our identification of the regularity properties of the functions in the completion of $W_{ \pm}$. As we have shown in the previous section that $K_{0} h^{\#}$ does not lie in the connected component of the identity of $\mathscr{G}$ it follows that $\phi$ lies in the connected component of the identity of $\mathcal{O}_{f}$ exactly when its pole in $\mathscr{N}$ is of even order. This argument proves:

Proposition 4.1. For each $\phi$ in $\mathscr{G}$,

$$
l(\phi)=\text { order of the pole of } \phi \text { in } \mathscr{N}(\bmod 2) .
$$

It would be interesting to devise a proof of this fact which did not appeal to the Hilbert space structure. One unnatural feature of the introduction of $\Gamma$ is that conjugation by $\Gamma$ does not leave $\mathscr{G}$ invariant. This seems especially unsatisfactory and it would be interesting to know whether there is any freedom in our choice of $\Gamma$ which would enable this to be overcome.

Our second by-product is:

Proposition 4.2. There is a projective representation $\rho$ of $\mathscr{G}$ by unbounded but densely defined operators on a dense (invariant) domain in the completion of $\wedge W_{+}$in its natural Hilbert space structure. The operators in this representation satisfy

$$
\rho(\phi) F(w) \rho(\phi)^{-1}=F(\phi w), \quad w \in W, \phi \in \mathscr{G} .
$$

We refer to $[\mathrm{CP}]$ for the proof of this result. It would be interesting to compute the two-cocycle on $\mathscr{G}$ which arises from this representation. In the genus one case it is computed (at the Lie algebra level) in [DJKM].

It is worth recording that one can define the operators $\rho(\phi)$ directly without recourse to the results of $[\mathrm{CP}]$. We will do this in the next section.

\section{The Projective Representation of $\mathscr{G}$}

It is possible to construct the operators $\rho(\phi)$ for $\phi$ in $\mathscr{G}$ which satisfy (4.1) by using some ideas from $[\mathrm{P}]$. We will not discuss the construction in complete generality but focus on particular elements of the group $\mathcal{O}_{f}$. Suppose that $G$ is in $\mathcal{O}_{f}$ and lies in the subset consisting of elements for which the kernel dimension of $Q_{-} G Q_{-}$ 
acting on $W_{-}$is zero. Then the operator

$$
R(G)=(G-1)\left(Q_{-} G+Q_{+}\right)^{-1}
$$

is well defined as $\left(Q_{-} G+Q_{+}\right)^{-1}$ exists precisely when $Q_{-} G Q_{-}$is invertible on $W_{-}$. In fact $R(G)$ is skew relative to the complex bilinear form on $W$. Introduce the notation

$$
R_{\delta \delta^{\prime}}=Q_{\delta} R(G) Q_{\delta^{\prime}} \quad\left(\delta, \delta^{\prime}= \pm\right)
$$

Now if $\delta \neq \delta^{\prime}$ then $R_{\delta \delta^{\prime}}$ has finite dimensional range and is skew relative to the bilinear form. Consider first the case of $R_{+-}$. Let $V_{+}$be the range of $R_{+-}$in $W_{+}$. Then one can find a finite dimensional subspace $V_{-}$of $W_{-}$whose dimension is the same as that of $V_{+}$and a complementary subspace $V_{-}^{\prime}$ of $W_{-}$such that $(v, w)=0$ for all $v$ in $V_{+}$and $w$ in $V_{-}^{\prime}$. Skew symmetry implies $R_{+}$is zero on $V_{-}^{\prime}$. A similar argument works for $R_{-}+$and so we may assume the existence of a finite dimensional subspace $W_{0}$ of $W$ on which the bilinear form is non-degenerate and a complementary subspace $W_{0}^{\prime}$ on which $R_{+-}+R_{-+}$is zero. Moreover we can find a basis of $W_{0}$ consisting of a basis $\left\{e_{j}\right\}$ of $W_{0} \cap W_{-}$and a dual basis $\left\{f_{j}\right\}$ of $W_{0} \cap W_{+}$.

Now introduce the elements $\Sigma_{j}\left(R_{+}-e_{j}\right) \wedge f_{j}$ and $\Sigma_{j}\left(R_{-+} f_{j}\right) \wedge e_{j}$ of $\wedge^{2} W$. We are then able to use the argument of Lemma 1.0 of $[\mathrm{P}]$ noting that while this result has only been formulated for finite dimensional $W$ trivial modifications allow us to extend the essential arguments to the case where $W$ is infinite dimensional provided we work only with elements of $\mathcal{O}_{f}$. Following $[\mathrm{P}]$ we define the following invertible elements of $\mathscr{C}(W)$ :

$$
\begin{aligned}
& g_{1}=\exp \left[\left(\Sigma_{j} c\left(R_{+-} e_{j}\right) c\left(f_{j}\right)\right) / 2\right], \\
& g_{2}=\exp \left[\left(\Sigma_{j} c\left(R_{-+} f_{j}\right) c\left(e_{j}\right)\right) / 2\right],
\end{aligned}
$$

and also the complex orthogonal:

$$
\left.G^{\prime}=\left(1+Q_{+} R\right) Q_{+}+\left(1-Q_{-} R\right)^{-1} Q_{-}\right)
$$

which commutes with $Q$. The group of operators commuting with $Q$ is represented on $\wedge W_{+}$by defining their action as

$$
\begin{aligned}
w_{1} \wedge w_{2} \wedge w_{3} \wedge \cdots \wedge w_{n} & \rightarrow G^{\prime} w_{1} \wedge G^{\prime} w_{2} \wedge G^{\prime} w_{3} \wedge \cdots \wedge G^{\prime} w_{n}, \\
\Omega & \rightarrow \Omega .
\end{aligned}
$$

We denote this operator by $\Gamma\left(G^{\prime}\right)$. Now define $\tilde{\Gamma}(G)$ by

$$
\tilde{\Gamma}(G)=F\left(g_{1}\right) \Gamma\left(G^{\prime}\right) F\left(g_{2}\right) .
$$

Some straightforward although lengthy calculations reveal as in $[\mathrm{P}]$ that

$$
\tilde{\Gamma}(G) F(w) \tilde{\Gamma}(G)^{-1}=F(G w) .
$$

In the general case where $Q_{-} G Q_{-}$may have a kernel a simple modification of this definition is required. We will discuss the case where $Q_{-} G Q_{-}$has a one dimensional kernel. If $v$ spans this kernel let $u=G v+x$, where $x$ is an element of $W_{-}$such that $(x, G v)=1$. (Recall that $W_{ \pm}$are isotropic with respect to the bilinear 
form so that $W_{-}$and $W_{+}$are dual to each other under the pairing induced by this form.) Now let $O(u)$ denote the operator $O(u) w=-w+(w, u) u$ and write $G_{0}$ for the product $O(u) G$. Let $w$ be an element of $W_{-}$in the kernel of $Q_{-} G_{0} Q_{-}$so that

$$
0=Q_{-} G_{0} w=Q_{-}(-G w+(G w, u) u) .
$$

Hence

$$
Q_{-} G Q_{-} w=(G w, u) x
$$

But

$$
\left(Q_{-} G Q_{-} w, G v\right)=(G w, G v)=(w, v)=0 .
$$

Combining the preceding two equalities gives $(G w, u)=0$ so that, in fact, $w$ is in the kernel of $Q_{-} G Q_{-}$. But

$$
Q_{-} G_{0} Q_{-} v=-G v+(G v, u) u=u-G v=x .
$$

Thus $w=0$ and $Q_{-} G_{0}+Q_{+}$is invertible. Thus we may define $\tilde{\Gamma}\left(G_{0}\right)$ as above and then define

$$
\tilde{\Gamma}(G)=F(u) \tilde{\Gamma}\left(G_{0}\right)
$$

It is easy to check that for all $w^{\prime}$ in $W$

$$
\tilde{\Gamma}(G) F\left(w^{\prime}\right) \tilde{\Gamma}(G)^{-1}=F\left(G w^{\prime}\right) .
$$

The case where the kernel dimension of $Q_{-} G Q_{-}$is greater than one is a little more complicated and we will not discuss it. (Provided one avoids the use of the complex conjugation in the way we have here one sees that all the main ideas are in $[\mathrm{P}]$.)

Proposition 5.1. The map sending $G$ to $\tilde{\Gamma}(G)$ is a projective representation of $\mathcal{O}_{f}$ on $\wedge W_{+}$.

Proof. The decomposition of the subset of $\mathcal{O}_{f}$ consisting of $G$ with $Q_{-} G+Q_{+}$ invertible defined in (5.1), (5.2) and (5.3) above shows in fact that each element of $\mathcal{O}_{f}$ decomposes into a product of elements which lie in subgroups on restriction to which $\tilde{\Gamma}$ is a representation. The projective character only enters when we try to combine them and a little thought reveals that the only place where the representation property fails is when we try to multiply elements of the form (5.1) by elements of the form (5.2). Now these are both in the so-called Clifford group (i.e. the group of invertible elements $g$ of the Clifford algebra which are such that $g c(w) g^{-1}=G w$ for some complex orthogonal $G$ on $W$ with $G-1$ finite rank). Now we are back in the finite dimensional case (cf. $[\mathrm{P}]$ and $[\mathrm{CP}]$ ) where we know that $\tilde{\Gamma}$ is a projective representation completing the proof.

Applying this to the example in Sect. 4 we obtain as an immediate corollary of this construction the fact that the operator $\tilde{\Gamma}\left(K_{0} h^{\#}\right)$ when applied to the cyclic vector $\Omega$ produces a vector which is in the odd sector of the Fock space (the completion of $\wedge W_{+}$). (The odd sector consists of eigenspaces of $N$ corresponding to odd integer eigenvalues.) Successive powers of this operator move one in and out of the odd sector. It is not surprising therefore to expect that the representation of $\mathscr{G}$ is at least cyclic if not irreducible in the Fock space. We do not have a proof of this conjecture in general, however in the genus one case a vertex operator 
construction exists which proves cyclicity and a weak form of the Boson-Fermion correspondence.

\section{The Boson-Fermion Correspondence and Vertex Operators}

The special property of the genus one case which enables us to take our arguments further is that the function $K_{0}$ has been given explicitly in [DJKM]. We let

$$
K_{q}(p)=c^{1 / 2}(h(p)-h(q)) /\left(k+k^{\prime}\right)(h(q))^{1 / 2},
$$

where $p$ is the point $(k, \omega), q$ is the point $\left(k^{\prime}, \omega^{\prime}\right)$ and $c=\left(a^{2}-b^{2}\right) / 4$ and the choice of square root is not important. What is special about the genus one case is that the point $q$ may be chosen arbitrarily whereas we had no control over the points $p_{1}, p_{2}, \ldots, p_{g}$ in the general case. The crucial fact is the

Lemma 6.1. Let $G$ be an element of $\mathcal{O}_{f}$ with the property that the kernel dimension of $Q_{-} G Q_{-}$is one. Suppose that $W_{-}=\operatorname{ker} Q_{-} G Q_{-} \oplus V$, where $V$ is a subspace on which $Q_{+} G Q_{-}$is zero. Then $\tilde{\Gamma}(G) \Omega=F(z) \Omega$ for some $z$ in $W_{+}$.

The proof depends on our construction of $G_{0}$ from $G$ in the previous section. Firstly we have $v$ spanning the kernel of $Q_{-} G Q_{-}$and $u=G v+x$. Then if $w$ is any multiple of $v$ :

$$
Q_{+} G_{0} Q_{-} w=-G w+(u, G w) G v=-G w+(x, G w) G v .
$$

The last two terms cancel because $(x, G v)=1$ so that $Q_{+} G_{0} Q_{-} w=0$. Now given the existence of $V$ as in the lemma we can write any $w$ in $W_{-}$as a sum $\lambda v+v_{1}$ with $v_{1}$ in $V$ and $G v_{1}$ in $W_{-}$. Thus,

$$
Q_{+} G_{0} Q_{-} w=Q_{+} G_{0} Q_{-} v_{1}=Q_{+}\left[-G v_{1}+\left(u, G v_{1}\right) u\right]=\left(u, G v_{1}\right) G v
$$

Now

$$
\left(u, G v_{1}\right)=\left(G v, G v_{1}\right)+\left(x, G v_{1}\right)=\left(v, v_{1}\right)=0,
$$

where we have used the isotropy of $W_{-}$. Hence we have $Q_{+} G_{0} Q_{-}=0$. A brief calculation then reveals that $Q_{+} R\left(G_{0}\right) Q_{-}=0$.

Now recall the construction of $\widetilde{\Gamma}\left(G_{0}\right)$ from the previous section. With

$$
R_{\delta \delta^{\prime}}=Q_{\delta} R\left(G_{0}\right) Q_{\delta^{\prime}}, \quad\left(\delta, \delta^{\prime}= \pm\right)
$$

we have

$$
\exp \left[\left(\Sigma_{j} c\left(R_{+-} e_{j}\right) c\left(f_{j}\right)\right) / 2\right]=1, \quad \exp \left[\left(\Sigma_{j} c\left(R_{-+} f_{j}\right) c\left(e_{j}\right)\right) / 2\right] \Omega=\Omega,
$$

and

$$
\Gamma\left[\left(1+Q_{+} R\right) Q_{+}+\left(1-Q_{-} R\right)^{-1} Q_{-}\right] \Omega=\Omega .
$$

Thus, $\tilde{\Gamma}\left(G_{0}\right) \Omega=\Omega$, implying that $\tilde{\Gamma}(G) \Omega=F(u) \Omega=F(G v) \Omega$. This completes the proof of the lemma.

The application of this result in the genus one case (and our failure in the higher genus case to see how to apply it) rests on our ability to show that there is a complex orthogonal consisting of multiplication by an element of $\mathscr{G}$ which satisfies the hypotheses of the lemma. In the genus one case this function is $\left(K_{q}\right)^{\#}$. 
To see that the lemma applies we note that $\vartheta\left(K_{q}\right)=q^{\#}-q+\infty_{-}-\infty_{+}$and that the kernel of $Q_{-}\left(K_{q}\right)^{\#} Q_{-}$is spanned by the function which is identically one. Hence for any $w$ in $W_{-}$we write

$$
w=[w-w(q)]+w(q) .
$$

Then $\left(K_{q}\right)^{\#}[w-w(q)]$ has no poles except at $\infty_{ \pm}$and so lies in $W_{-}$. Thus the subspace $V$ consists of the functions $w-w(q)$. We conclude then that

$$
\tilde{\Gamma}\left(\left(K_{q}\right)^{\#}\right) \Omega=F\left(\left(K_{q}\right)^{\#}\right) \Omega .
$$

A formal expression for $\tilde{\Gamma}\left(\left(K_{q}\right)^{\#}\right)$ is given in [DJKM] which for those familiar with these matters will make it clear why one should think of it as a vertex operator.

To see why (6.1) implies both cyclicity of the representation of $\mathscr{G}$ and a weak form of the Boson-Fermion correspondence we need one more property of $K_{q}$. This is the observation of [DJKM] that the function $q \rightarrow K_{q}\left(p^{\#}\right)$ acts as a Cauchy kernel for the path $\gamma$ in the sense that, as one easily calculates,

$$
Q_{+} w(q)=\int_{\gamma} w(p) \sqrt{c h(p)} K_{p}\left(q^{\#}\right) \frac{d k}{2 \pi i \omega},
$$

where $q$ lies on the $\infty_{ \pm}$side of $\gamma$ and $p$ has coordinates $(\omega, k)$. We now appeal to the theory of Fock representations of the Clifford algebra [A] to see that $q \rightarrow F\left(K_{q}^{\#}\right) \Omega$ is weakly continuous. So we may form the Bochner integral:

$$
\int_{\gamma} w(q) \sqrt{\operatorname{ch}(q)} F\left(\left(K_{q}\right)^{\#}\right) \Omega \frac{d k^{\prime}}{2 \pi i \omega^{\prime}}
$$

for $w$ in $W_{+}$where $q$ has coordinates $(\omega, k)$. By virtue of the Cauchy kernel property this integral defines an element of the Fock space equal to $F(w) \Omega$. Hence we can span the subspace of the Fock space corresponding to the eigenvalue one of $N$ by using vectors constructed as weak limits of linear combinations of the operators representing the group $\mathscr{G}$. This argument can now be repeated. To get the eigenspace of $N$ corresponding to the eigenvalue 2 we consider for $w$ in $W_{+}$,

$$
\tilde{\Gamma}\left(\left(K_{q}\right)^{\#}\right) F\left(K_{q} w\right) \Omega=F(w) F\left(\left(K_{q}\right)^{\#}\right) \Omega .
$$

Now it is clear how to construct any vector in $W_{+} \wedge W_{+}$by repeating the previous argument. In this way we build up the Fock space cyclically from the vector $\Omega$, using the projective action of $\mathscr{G}$. We summarise these arguments as:

Proposition 6.2. The projective representation of $\mathscr{G}$ on the Fock space (the completion of $\left.\wedge W_{+}\right)$is cyclic with cyclic vector $\Omega$ in the genus one case. The action of the element $\left(K_{q}\right)^{\#}$ on $\Omega$ gives a vector in the eigenspace of $N$ corresponding to the eigenvalue one. Eigenspaces corresponding to larger eigenvalues may be constructed by an inductive procedure.

This result can be interpreted as a form of the Boson-Fermion correspondence as we shall now explain. Note firstly that if $f$ is a meromorphic function on $\mathscr{M}$ which is regular on $\mathscr{N}-\left\{\infty_{+}, \infty_{-}\right\}$and satisfies $f^{\#}=-f$ then $(\exp f)^{\#}=$ $(\exp f)^{-1}$ so that $\exp f$ is a complex orthogonal. However, it is not straightforward to determine when $\exp f$ is in $\mathscr{G}$. In fact we have not considered the appropriate 
Lie structure for $\mathscr{G}$ at all. Rather than address this question directly it is sufficient for our purposes to note that there are many such $f$ with the property that $Q_{\delta} f Q_{-\delta}$ is finite rank and hence by general arguments $\left[\mathrm{CHO}^{\prime} \mathrm{B}\right] \exp f$ lies in the restricted orthogonal group. We denote the set of all such meromorphic $f$ by $\mathscr{L}$.

By general arguments $[\mathrm{L}, \mathrm{CR}]$ the 2-cocycle for our projective representation of the restricted orthogonal group defines, a projective action of this Lie algebra $\mathscr{L}$, where the Lie algebra cocycle is:

$$
\sigma(f, f)=\operatorname{tr}\left(Q_{-} f Q_{+} f^{\prime} Q_{-}-Q_{-} f^{\prime} Q_{+} f Q_{-}\right) .
$$

(We do not have a method for explicitly computing this cocycle in general although [DJKM] give it in the genus one case.) Denote the projective Lie algebra action by $f \rightarrow \rho^{\prime}(f)$ and consider the commutation relations satisfied by the operators $\rho^{\prime}\left(f_{1}\right)$ and $\rho^{\prime}\left(f_{2}\right)$ for $f_{1}$ and $f_{2}$ in $\mathscr{L}$ :

$$
\left[\rho^{\prime}\left(f_{1}\right), \rho^{\prime}\left(f_{2}\right)\right]=\sigma(f, f) I,
$$

where $I$ is the identity operator. Those readers still with us will recognise these as Boson commutation relations. Thus the Lie algebra $\mathscr{L}$ is a "Boson algebra," the operators $\rho(\varphi)$ for $\varphi$ in $\mathscr{G}$ are generalised Weyl operators and we have shown above that associated with our representation of the Clifford algebra (the fermions) there is a representation of a Boson algebra and that the Fermions may be recovered from this boson representation via Proposition 6.2.

This is what we earlier referred to as a weak form of the Boson-Fermion correspondence. It contrasts with the situation in the theory of loop group representations in that there one may construct the elements of the Clifford algebra as limits, in the sense of strong convergence on a dense domain, of complex multiples of the representers of certain loop group elements (see [CR]). Here we have only been able to show that one can construct the vectors in $\wedge W_{+}$of the form

$$
F\left(w_{1}\right) F\left(w_{2}\right) \cdots F\left(w_{n}\right) \Omega
$$

by using the representers of "loops" in $\mathscr{G}$.

\section{The Landau-Lifshitz Equation}

In [SW] Segal and Wilson elaborate the strategy of M. and Y. Sato which connects a certain infinite dimensional Grassmannian of subspaces of a complex Hilbert space with solutions of the $\mathrm{KdV}$ equation and related hierarchies of soliton equations. Our analysis here was partly motivated by their paper and indeed we expect similar results to hold in the context of the Landau-Lifshitz equation. Firstly we note that, following [DJKM], we need to consider a matrix valued version of our group obtained by tensoring onto our line bundle a copy of $\mathbb{C}^{2}$. Recalling Remark 3.4 we consider the group $\mathscr{G}_{2}$ of $2 \times 2$ matrix valued meromorphic functions on $\mathscr{M}$ which are holomorphic on $\mathscr{N}-\{z, w\}$ and satisfy $\varphi^{\#}=\left(\varphi^{-1}\right)^{\tau}$, where $\tau$ denotes the matrix transpose. We continue to denote our space of sections of the resulting bundle by $W$ and the splitting by $W=W_{-} \oplus W_{+}$. Then this group also has the property that it is a subgroup of the group $\mathcal{O}_{f}$ defined by this splitting. 
Now in this setting there is a "real" Grassmannian consisting of subspaces which are "close" to $W_{-}$in the sense that the orthogonal projections onto these subspaces differ by say a trace class operator. One might anticipate therefore that the appropriate adaptation of [SW] would involve associating solutions of the Landau-Lifshitz equation with certain elements of this Grassmannian. At first sight [DJKM] is of no help here because their method of constructing solutions involves introducing singular functions (actually distributions) on $\mathscr{M}$ and treating them as if they were elements of the group $\mathscr{G}_{2}$. Thus they write down formal expressions for the operators which represent these singular objects and allow them to act on the vacuum or cyclic vector. They then compute various matrix elements which depend on the flow parameters of the Landau-Lifshitz hierarchy thus obtaining their tau function and solutions. Now these calculations are difficult to make precise as at best the "operators" under consideration are densely defined quadratic forms on the Fock space. However there is an alternative strategy which is suggested by the form of the solutions appearing in [DJKM]. When one considers the Lax form of the Landau-Lifshitz equation one sees that the solutions in this case are constructed in [DJKM] using bona fide elements of $\mathscr{G}_{2}$. As $\mathscr{G}_{2}$ acts on our Grassmannian it seems plausible to seek a connection between points in the Grassmannian and solutions of the Lax form via the construction of these elements of $\mathscr{G}_{2}$. One can get a hint of how this works from Eq. (3.10) in [DJKM] which constrains the elements of $\mathscr{G}_{2}$ needed to construct solutions via an isotropy condition arising from the splitting $W=W_{-} \oplus W_{+}$. What is currently absent is an understanding of the meaning or origin of these elements which is analogous to the more detailed understanding of the corresponding construction for $\mathrm{KdV}$ in [SW].

Remark. There is another approach to the Landau-Lifshitz equation described in [R]. At first sight one might expect that it is related to the approach described above as both exploit a splitting of a space of meromorphic functions on an elliptic curve. However as yet we see no link at all between them.

Acknowledgements. The first and third named authors thank Dai Evans for invitations to participate in the Warwick Symposium on Operator Algebras and Applications where this work was begun. We have discussed various aspects of this paper with many people but special thanks are due to John Palmer, John Jones and Roger Richardson for their assistance. The third named author acknowledges with gratitude the financial support of the Australian Research Council and the hospitality of the Department of Pure Mathematics as the University of Adelaide in the final stages of this project.

\section{References}

[A] Araki, H.: On quasifree states of the CAR and Bogoliubov automorphisms. Publ. RIMS 6, 385-442 (1971)

[C] Clemens, C. H.: A scrap book of complex curve theory. New York: Plenum Press 1980

[CP] Carey, A. L., Palmer, J.: Infinite dimensional spin and complex orthogonal groups. J. Funct. Anal. 83, 1-43 (1989)

[CR] Carey, A. L., Ruijsenaars, S. N. M.: On fermion gauge groups, current algebras and Kac-Moody algebras. Acta Applicandae Math 10, 1-86 (1987)

[CHO'B] Carey, A. L., Hurst, C. A., O'Brien, D. M.: Automorphisms of the canonical anticommutation relations and index theory. J. Funct. Anal. 48, 360-393 (1982) 
[DJKM] Date, E., Jimbo, M., Kashiwara, M., Miwa, T.: Landau-Lifshitz equation: Solitons, quasi-periodic solutions and infinite dimensional Lie algebras. J. Phys. A, 16, 221-236 (1983)

[D] Date, E.: On Landau-Lifshitz equation and infinite dimensional groups. In: Infinite dimensional groups with applications. M.S.R.I. Publ. vol. 4, pp 71-82. Berlin, Heidelberg, New York: Springer 1985

[G] Gunning, R. C.: Lectures on Riemann surfaces. Princeton Mathematical Notes. Princeton, NJ: Princeton University Press 1966

[L] Lundberg, L.-E.: Quasi-free second quantisation. Commun. Math. Phys. 50, 103-112 (1976)

[P] Palmer, J.: Products in spin representations. Adv. App. Math. 2, 290-326 (1981)

[R] Rodin, Y.: The Riemann boundary problem on Riemann surfaces and the inverse scattering problem for the Landau-Lifshitz equation. Physica 11D, 90-108 (1984)

[SW] Segal, G. B., Wilson, G.: Loop groups and equations of KdV type. Publications IHES 61, 5-65 (1985). For more information on Grassmanians and related matters see Pressley, A. N., Segal, G. B. (eds.). Loop groups. Oxford: Oxford University Press 1986

Communicated by H. Araki

Received September 13, 1988; in revised form November 1, 1989 